\title{
Alteraciones de la huella plantar en preescolares del Centro Infantil del Buen Vivir "Fran- cisco Chiriboga"
}

Alterations of the footprint in preschoolers of the Center for Children of Good Living "Francisco Chiriboga"

\author{
Autores: \\ Valeria Maribel Motoche Santos ${ }^{1}$ \\ Bárbara Leyanis Nuñez Sánchez ${ }^{1}$ \\ Laura Verónica Guaña Tarco ${ }^{1}$ \\ René Yartu Couceiro ${ }^{1,2}$ \\ Adriana Elizabeth Oleas López ${ }^{3}$
}

${ }^{1}$ Universidad Nacional del Chimborazo, Riobamba-Ecuador.

${ }^{2}$ Hospital Pediátrico Alfonso Villagómez en Riobamba, Riobamba-Ecuador.

${ }^{3}$ Instituto Superior Tecnológico Riobamba, Riobamba-Ecuador.

Autor de correspondencia: Bárbara Leyanis Nuñez Sánchez. Universidad Nacional de Chimborazo, Ave. Antonio José de Sucre Km 1 1/2, Riobamba, Ecuador. Email: bnunez@unach.edu.ec. Teléfono: 0998418983.

\section{RESUMEN}

La forma en que se manejan los problemas biomecánicos en niños constituye un tema controversial en el campo de la rehabilitación física. Se desarrolló un proceso investigativo con el objetivo de: describir la evolución de un grupo de pacientes pediátricos mayores de dos años diagnosticados con pie plano atendidos en consulta de fisioterapia en la ciudad de Riobamba; la que fue de tipo observacional descriptivo, de corte longitudinal, cuya población de estudio estuvo constituida por las fichas de control, evaluación y valoración de 30 niños mayores de 2 años que acudieron a consulta de fisioterapia acompañados de un tutor legal por presentar signos de alteraciones de la huella plantar (pie plano). Se observó un predominio de los pacientes con edades comprendidas entre cuatro y cinco años, para un 33 y $27 \%$ respectivamente. El 93\% de los participantes presentó alteraciones de la huella plantar comprobada mediante medición utilizando el plantígrafo, pero el 53\% tuvo pie plano fisiológico. Luego de aplicados los ejercicios de Risser, los datos reflejaron una elevación de la cifra de huella plantar normal hasta el 33\% y una disminución en el grado de afectación en el 35\% de los niños diagnosticados. Los resultados de la recuperación fueron ratificados mediante el análisis del desgaste del calzado en los pacientes pediátricos en cuestión.

Palabras clave: pie plano, niño, fisioterapia, terapia por ejercicio. 


\begin{abstract}
The way how biomechanical problems are handled is a controversial topic in the field of physical rehabilitation. This is a research was carried out in order to describe the evolution of a group of pediatric patients over two years of age diagnosed with flat feet treated in a physiotherapy consultation in the city of Riobamba. This research was of observational descriptive type and longitudinal-cut. The study population consisted of the control, evaluation and assessment cards of 30 children over 2 years of age who attended a physiotherapy consultation accompanied by a legal guardian to show signs of alterations in the footprint (flat foot). A predominance of patients between four and five years of age with $33 \%$ and $27 \%$ respectively was observed. $93 \%$ of the participants presented alterations of the footprint checked by the means of a machine for measuring foot plant, but 53\% had physiological flat feet. After applying the Risser exercises, the data was reflected in the figure of normal footprint up to $33 \%$, and a decrease of affectation in $35 \%$ of the children diagnosed. The results of the recovery were confirmed through the analysis of footwear wear in the pediatric patients in question.
\end{abstract}

Keywords: Flatfoot, Child, exercises, Physical Therapy Specialty, Exercise Therapy.

\title{
INTRODUCCIÓN
}

El aspecto nutricional constituye un pilar importante en el adecuado desarrollo del niño. Durante la infancia, la realización de ejercicio físico reduce los factores de riesgo ante diferentes enfermedades. El pie resulta la porción terminal de una extremidad inferior cuyas funciones principales es sostener el peso del cuerpo y permitir la locomoción. Las alteraciones de esta estructura anatómica deben ser atendidas desde etapas tempranas de la vida. ${ }^{(1)}$

Con frecuencia, el desconocimiento de los padres de familia con respecto a la correcta postura corporal de los infantes, resulta la principal causa por la que no se recibe la debida atención facultativa oportunamente; lo que ocasiona que en la etapa adulta sean frecuentes alteraciones ortopédicas difíciles de corregir y que limitan la movilidad y las actividades de la vida diaria del individuo. ${ }^{(2)}$

Este tipo de problemas puede ser corregido tempranamente mediante la adecuada intervención fisioterapéutica. ${ }^{(3)}$ El pie plano constituye una de las noxas ortopédicas más frecuentes en el paciente pediátrico; el tratamiento rehabilitador al respecto busca fortalecer los músculos de la región plantar y dar tono a la fascia; lo que da lugar a la formación del arco longitudinal externo y principalmente el interno. ${ }^{(4,5)}$

Cuando las deformidades del pie persisten hasta la edad adulta, su corrección se torna prácticamente imposible; En cuyo caso, solo se puede disminuir notablemente la sintomatología a través de tratamientos. ${ }^{(6)}$ 
En relación con lo antes expuesto, se desarrolló un proceso investigativo con el objetivo de: describir la evolución de un grupo de pacientes pediátricos mayores de dos años diagnosticados con pie plano atendidos en consulta de fisioterapia en la ciudad de Riobamba.

\section{MATERIAL Y MÉTODOS}

Se realizó un estudio observacional descriptivo, de corte longitudinal; en el que la población de estudio estuvo constituida por las fichas de control, evaluación y valoración de 30 niños mayores de 2 años que acudieron a consulta de fisioterapia acompañados de un tutor legal por presentar signos de alteraciones de la huella plantar (pie plano).

Los datos fueron recolectados mediante la técnica de revisión de documentos (ficha de control, evaluación y valoración del paciente); lo que permitió recolectar la cifras relacionadas con la valoración en las afectaciones de la huella plantar, que luego fueron organizadas en una base de Microsoft Excel.

El procesamiento estadístico de los datos se realizó utilizando técnicas del nivel descriptivo mediante análisis de frecuencias relativas y absolutas; lo que facilitó la posterior presentación en tablas y gráficos que facilitaron su interpretación y el arribo a conclusiones.

Durante la realización del estudio se tuvo en cuenta el cumplimiento de los principios bioéticos. Se respetó la autonomía de los involucrados al solicitar el consentimiento informado de los tutores legales de los menores. La dirección del centro educativo brindó la respectiva autorización. Los datos relativos a cada paciente resultaron de uso confidencial, no existiendo uso maleficente de los mismos.

\section{RESULTADOS Y DISCUSIÓN}

Los datos recogidos en ficha de control, evaluación y valoración del paciente, relacionados con la medición de la huella plantar mediante el plantígrafo, permitieron apreciar que las alteraciones presentes correspondían con el pie plano en sus diferentes variantes. Los resultados arrojaron un predominio del género femenino (67\%) entre la población de estudio; al respecto Ezema et al. ${ }^{(7)}$ observaron resultados similares en su proceso investigativo.

Gráfico 1. Distribución de la población de estudio según la edad

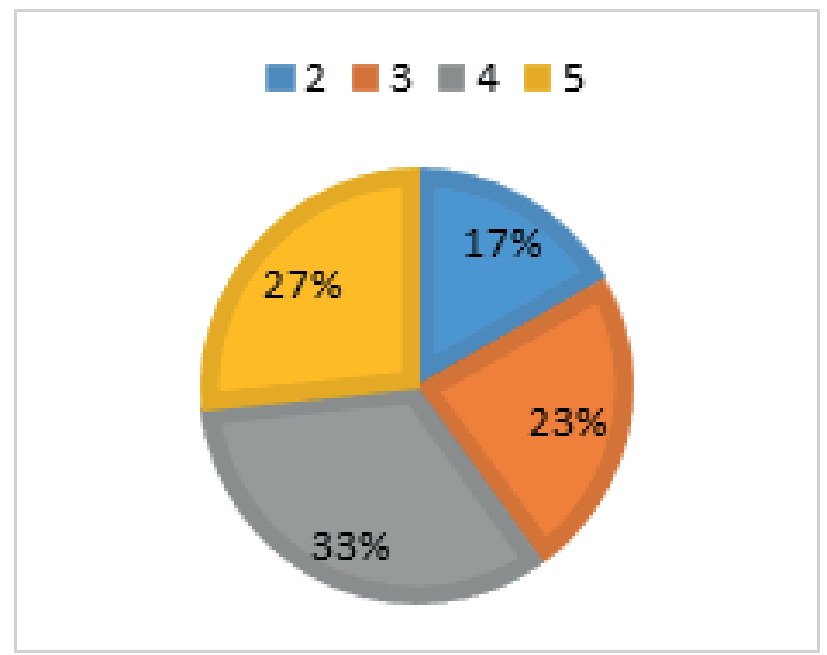


En la población de estudio predominaron los pacientes con edades comprendidas entre cuatro y cinco años, para un 33 y 27\% respectivamente (gráfico 1). En relación con ese resultado, Alvi y Wilson ${ }^{(8)}$ plantean que la detección y tratamiento fisioterapéutico del pie plano desde el momento en que el infante inicia la deambulación, resulta de vital importancia en el alcance del efecto deseado; atendiendo a lo cual, hubiera sido preferible que la mayoría de los pacientes tuvieran el diagnóstico definitivo entre los dos y tres años de edad.

Gráfico 2. Distribución de la población de estudio según alteración de la huella plantar comprobada

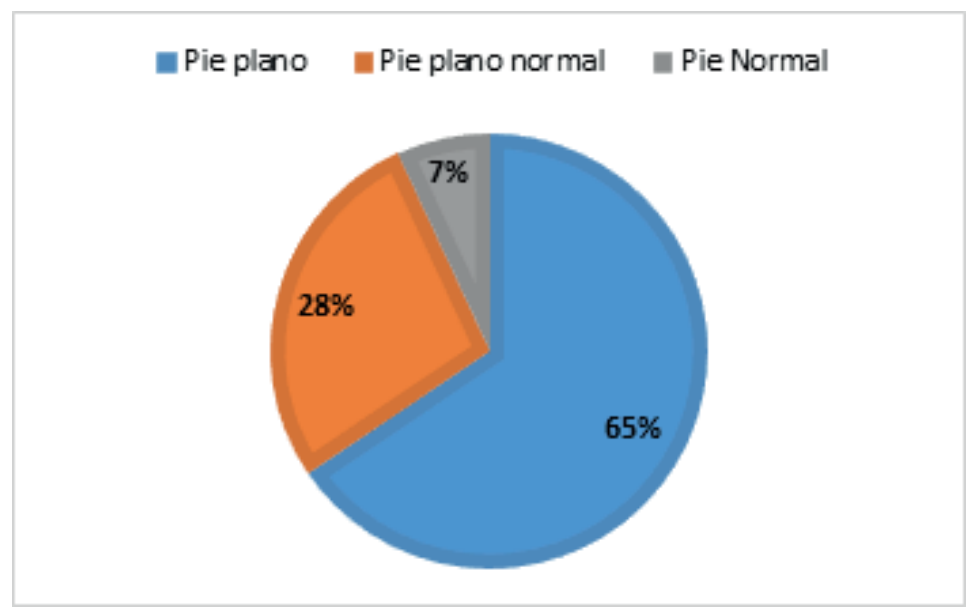

Los datos relacionados a las mediciones de la huella plantar durante el diagnóstico permitieron establecer que el $93 \%$ de los pacientes pediátricos presentó alteraciones al respecto; la mayoría de estos en la categoría de pie plano. (gráfica 2).

Al respecto, Halabchi et al. ${ }^{(9)}$ plantean que el estudio de esta patología en la edad pediátrica resulta importante, para elevar las posibilidades de éxito en su corrección mediante el tratamiento adecuado.

Gráfico 3. Tipo de alteración de la bóveda plantar en la población de estudio

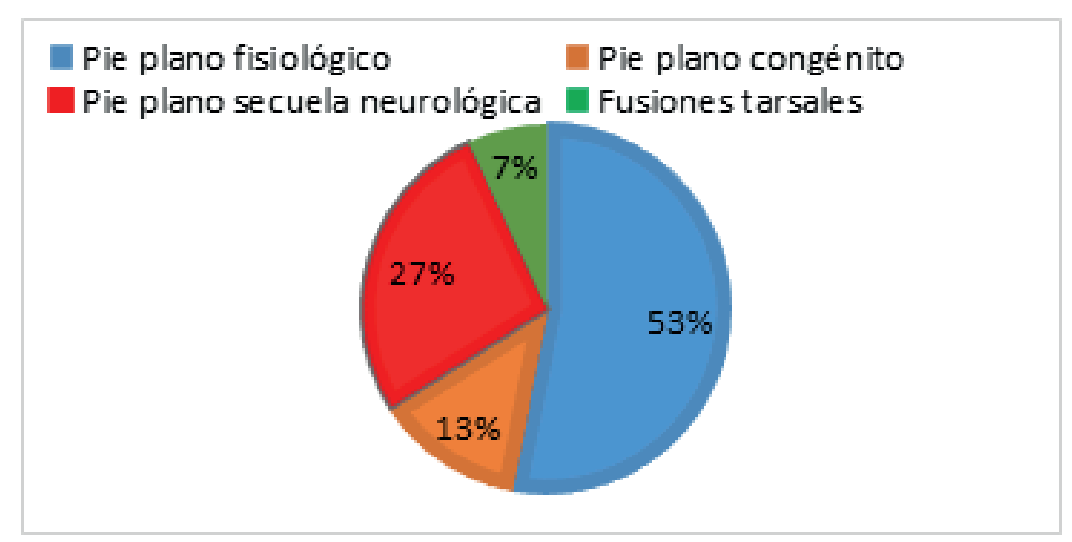

En relación con el tipo de alteración en la bóveda plantar, los investigadores observaron que el $53 \%$ de los pacientes pediátricos presentó pie plano fisiológico, el que es propio de la edad debido a la presencia de tejido adiposo en esa región (gráfica 3).

En un estudio similar realizado por Zárate Barchello et al., ${ }^{(10)}$ ellos detectaron una situación similar; además de sugerir la adopción de un conjunto de medidas terapéuticas que abarcan 
desde el uso de plantillas correctoras hasta la cirugía correctora de músculos y huesos que construyen la bóveda plantar; la aplicación de una u otra dependerá del tipo de alteración y la edad del sujeto afectado.

Gráfico 4. Evolución de los pacientes luego de tratamiento fisioterapéutico

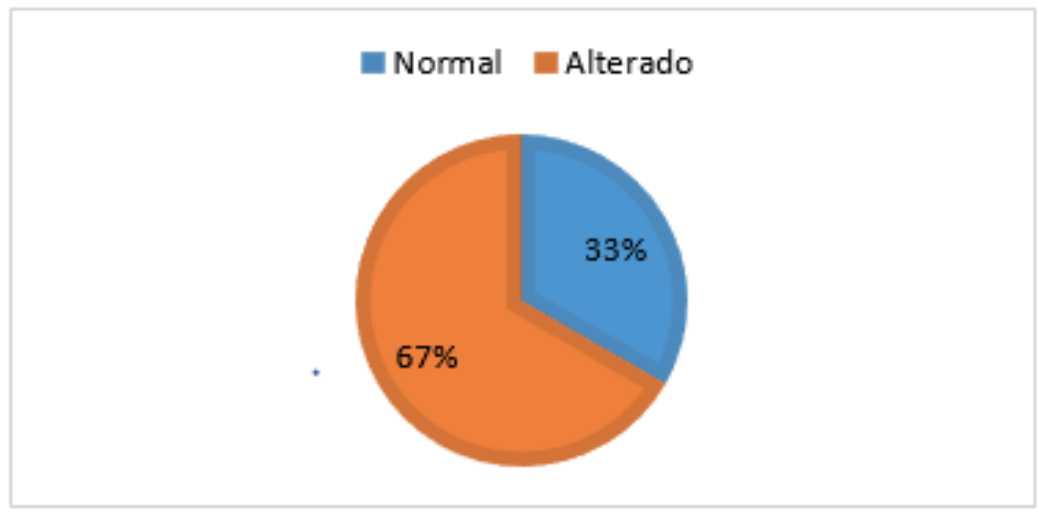

Los documentos revisados reflejaron que los niños y niñas afectados fueron tratados mediante los ejercicios de Risser. La valoración de la evolución de los pacientes, al momento de recoger los datos, reportó una elevación de la cifra de huella plantar normal hasta el 33\% (gráfico 4). Salazar y Magali ${ }^{(11)}$ analizaron la composición de la huella plantar en niñas y niños con edades comprendidas entre 4 y 10 años, obteniendo resultados similares a los de la presente investigación.

En la ficha de control, evaluación y valoración del paciente se refiere que la valoración de la evolución de la huella plantar fue corroborada mediante el test de Hernández Corvo; lo que evidenció la recuperación del $26 \%$ de los pacientes y que se redujo el grado de afectación en el $35 \%$.

Autores como Claure et al. ${ }^{(12)}$ describen la fiabilidad del Test de Hernández Corvo para valorar las afectaciones por pie plano; mientras que, $\operatorname{Keer}^{(13)}$ enfatiza en las potencialidades la fisioterapia en rehabilitación de afecciones por pie plano.

Gráfico 5. Valoración del desgaste del calzado como parte de la evolución de los pacientes de la población de estudio

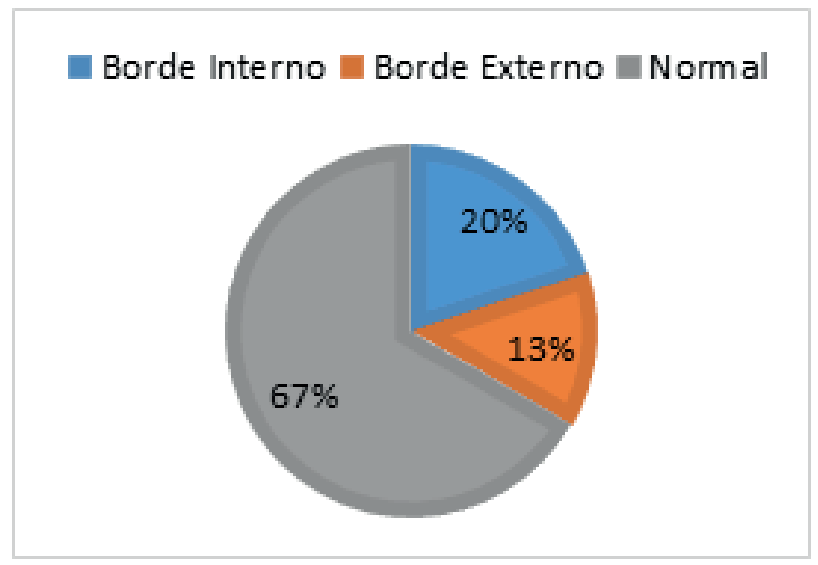


Otro resultado favorable en cuanto a la recuperación de los pacientes pediátricos de la población de estudio estuvo referido al análisis del desgaste del calzado; en el que se observó que el 67\% fue catalogado como normal (gráfico 5).

Al respecto, Arroyo, ${ }^{(13)}$ en su proceso investigativo, tomó el desgaste del calzado como un indicador de la presencia de afecciones de la huella plantar en los niños que participaron en ese estudio.

Conflictos de intereses: los autores declaran que no existen.

Declaración de contribuciones: Valeria Maribel Motoche Santos trabajó en la generalidad de las etapas del proceso investigativo, Bárbara Leyanis Nuñez Sánchez y Laura Verónica Guaña Tarco realizaron asesoría metodológica durante proceso investigativo y participaron en la redacción del artículo, René Yartu Couceiro y Adriana Elizabeth Oleas López trabajaron en la búsqueda bibliográfica y en la redacción final del artículo.

\section{CONCLUSIONES}

En la población de estudio predominaron los pacientes con edades comprendidas entre cuatro y cinco años; así como aquellos que presentaron alteraciones de la huella plantar comprobada mediante medición utilizando el plantígrafo, y entre estos últimos los que tuvieron pie plano fisiológico.

Luego de aplicados los ejercicios de Risser, los datos reflejaron una elevación de la cifra de huella plantar normal entre los participantes y una disminución en el grado de afectación en los niños diagnosticados; los que fueron ratificados mediante el análisis del desgaste del calzado.

\section{REFERENCIAS BIBLIOGRÁFICAS}

1. Justo Martínez E. Desarrollo psicomotor en educación infantil: Bases para la intervención en psicomotricidad. Almería: Universidad de Almería; 2014.

2. Sielatycki JA, Hennrikus WL, Swenson RD, Fanelli MG, Reighard CJ, Hamp JA. In-Toeing is Often a Primary Care Orthopedic Condition. The J of Pediatrics [Internet]. 2016 Oct [citado 2018 Ene 11]; 177: 297-301. Disponible en: https://www.jpeds.com/article/S0022-3476(16)30382-1/fulltext. https://doi.org/10.1016/j.jpeds.2016.06.022.

3. Drefus LC, Kedem P, Mangan SM, Scher DM, Hillstrom HJ. Reliability of the Arch Height Index as a Measure of Foot Structure in Children. Pediatric Physical Therapy [Internet]. 2017 [citado 2018 Ene 15]; 29(1): 83-88. Disponible en: https://journals.lww.com/pedpt/fulltext/2017/01000/Reliability_of_the_Arch_Height_Index_as_a_Measure.24.aspx. doi: 10.1097/PEP.0000000000000337. 
4. Schreiber S, Parent EC, Moez EK, Hedden DM, Hill DL, Moreau M, Southon SC. (2016). Schroth Physiotherapeutic Scoliosis-Specific Exercises Added to the Standard of Care Lead to Better Cobb Angle Outcomes in Adolescents with Idiopathic Scoliosis-an Assessor and Statistician Blinded Randomized Controlled Trial. Scoliosis and Spinal Disorders [Internet]. 2017 Dic [citado 2018 Ene 25]; 12(26): 1-8. Disponible en: https://link.springer.com/content/pdf/10.1186\%2Fs13013-017-0137-8.pdf. DOI 10.1186/s13013-017-0137-8.

5. Barroso-Planes I, Garcés-Carracedo JE, Padilla-Dip R. Ejercicios físicos terapéuticos para el tratamiento de deformidades ortopédicas de pies planos y escoliosis en escolares con retraso mental severo. OLIMPIA [Internet]. 2017 [citado 2018 Ene 12]; 14(42): 75-86, 75-86. Disponible en: https://dialnet.unirioja.es/descarga/articulo/6210629.pdf.

6. Motoche-Santos VM. Tratamiento fisioterapéutico preventivo de la huella plantar en niños a partir de 2 años de edad que asisten al centro infantil del buen vivir "Francisco Chiriboga" febrero-julio 2016 [Tesis de grado]. Riobamba: Universidad Nacional de Chimborazo; 2017.

7. Ezema CI, Abaraogu UO, Okafor GO. Flat foot and associated factors among primary school children: A cross-sectional study. Hong Kong Physiotherapy J [Internet]. 2014 [citado 2018 Ene 24]; 32(1): 13-20. Disponible en: https://www.sciencedirect.com/science/article/pii/S1013702513000493. https://doi.org/10.1016/j.hkpj.2013.05.001.

8. Alvi F, Wilson N. Evidence-Based Treatment of Flexible Flat Foot in Children. En: Paediatric Orthopaedics. Cham: Springer International Publishing; 2016. p. 171-174.

9. Halabchi F, Mazaheri R, Mirshahi M, Abbasian L. Pediatric flexible flatfoot; clinical aspects and algorithmic approach. Iran J Pediatr [Internet]. 2013 Jun [citado 2018 Ene 22] ;23(3): 247-60. Disponible en: https://www.ncbi.nlm.nih.gov/pmc/articles/PMC3684468/.

10. Zárate Barchello A, Pereira López MA, Ibarrola Zárate J, Kikuchi A, Sanabria L. Prevalencia de pie plano en niños escolares de Asunción y Gran Asunción, en el año 2008. An. Fac. Cienc. Méd. (Asunción) [Internet]. 2009 Dic [citado 2018 Feb 9]; 42(2): 13-18. Disponible en:

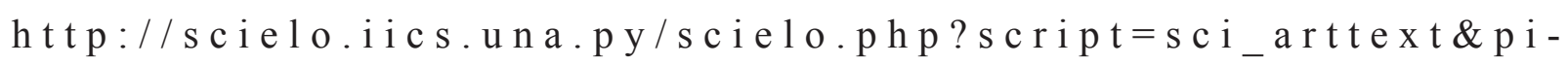
$\mathrm{d}=\mathrm{S} 1816-89492009000200002 \& \operatorname{lng}=\mathrm{es}$.

11. Salazar M, Magali R. (2015). Ejercicios de Risser en niñas y niños de 4 a 10 años que presentan pie plano de grado I y II en la Unidad Educativa Rosa Zarate de la ciudad de Salcedo [Tesis de grado]. Ambato: Universidad Técnica de Ambato; 2015.

12. Claure JC, Pedraza PA, Fernandez SP, Ortiz A. Métodos alternativos de evaluación en niños de 4 - 6 años con pie plano 1. Univ. Cienc. Soc. [Internet]. 2014 Dic [citado 2018 Feb 2]; (13): 19-25. Disponible en: http://www.revistasbolivianas.org.bo/scielo.php?script=sci_arttext\&pid=S8888-88882014000200004\&lng=en. 
13. Keer RJ. The Role of Physiotherapy in Marfan Syndrome. En: Diagnosis and Management of Marfan Syndrome. London: Springer; 2016. p. 169-174.

14. Arroyo, M. Detección precoz de patologías de pie en la infancia y su abordaje kinésico [Tesis de grado en Internet]. Mar del Plata: Universidad FASTA; 2013. [citado 2018 Feb 5]. Disponible en: http://redi.ufasta.edu.ar:8080/xmlui/bitstream/hand1e/123456789/104/2013_K_003.pdf?sequence=1.

Recibido: 15 de marzo de 2018 Aprobado: 05 de junio de 2019 